\title{
Structural Implications of Universal Complementarities in Translation-High Accuracy at the Decoding Site
}

\author{
Kozo Nagano \\ Nagano Research Institute of Molecular Biology, Yokohama, Japan \\ Email: fwkz4754@nifty.com
}

Received 26 November 2014; accepted 12 April 2015; published 17 April 2015

Copyright @ 2015 by author and Scientific Research Publishing Inc.

This work is licensed under the Creative Commons Attribution International License (CC BY).

http://creativecommons.org/licenses/by/4.0/

(c) (1) Open Access

\begin{abstract}
X-ray structures of transfer RNAs (tRNAs) bound to the whole ribosome do not fully explain the mechanism of translation. The cause of the failure seems to come mainly from a high $\mathrm{Mg}^{2+}$ ion concentration compared to that in the living cells. There exists a wide range of nucleotide sequence conservation in tRNA and ribosomal RNAs (rRNAs) of small and large subunits as well as sequence complementarities that seems to explain how high accuracy in translation can be achieved at the decoding site. Conformational transition between U33-folded and U33-extended forms of anticodon loops of tRNAs and G-C pair formation and disruption between C1399 and G1504 of 16S rRNA, etc. play the central role in explaining why E-site tRNA can automatically be expelled when an aminoacyl-tRNA at the $A$ site turns out to be cognate.
\end{abstract}

\section{Keywords}

Ribosomal RNA, Transfer RNA, Spectinomycin, Universal Rule, U33-Folded Form, U33-Extended Form, GTP Hydrolysis, Cognate, Near-Cognate

\section{Introduction}

Protein synthesis is carried out on the ribosome that is composed of small and large subunits. Amino acid is brought into the interface of the two subunits as a form of aminoacyl-tRNA (aa-tRNA) in cooperation with elongation factor $\mathrm{Tu}(\mathrm{EF}-\mathrm{Tu})$, complexed with guanosine triphosphate (GTP). Through the intersubunit space tRNAs move along with message-carrying RNA (mRNA) from the entrance toward the exit. There exist three sites for the tRNA-mRNA complex; the first site is for an aa-tRNA (A site), the second for a peptidyl-tRNA (P site), and the third for the exit of a deacylated tRNA (E site). The peptidyl group of P-site tRNA is transferred to 
the aminoacyl group of aa-tRNA at the A site on the peptidyl transferase center (PTC). The peptidyl-tRNA on the A site and the deacylated tRNA on the P site are forced to move in the next step toward the P and E sites, respectively, by a mechanism called translocation. During the course of translocation, it is known that the classical state of two tRNAs, A/A and P/P state, passes through an intermediate state, A/P and P/E, that is called hybrid state, and finally reaches the classical P/P and E/E state. X-ray crystallographic analyses have clarified the three dimensional (3D) structure of Thermus thermophilus $70 \mathrm{~S}$ ribosome at $5.5 \AA$ resolution that contained bound mRNA of only A- and P-site codons and three deacylated tRNAs bound to A, P, and E sites [1]. Successively, the structure of 70S ribosome containing a model of mRNA and two tRNAs bound to P and E sites was solved at $3.7 \AA$ resolution [2]. It was shown that the adenine base at the CCA end of the deacylated tRNA bound to the E site is recognized by C2394 and G2421 of helix 88 of 23S rRNA (H88) [3]. Nucleotide numberings of $16 S$ and 23S rRNAs follow the convention of [4], while helix numberings of 16S and 23S rRNAs that of [5]. On the other hand, the T-arm, D-arm, and the 3'-acceptor arm of A-site tRNA in the X-ray structure of the 70S ribosome were invisible, while an unintentional E-site tRNA was observed along with P-site tRNA complexed with mRNA [6]. Base pairing of the E-site codon to the anticodon of E-site tRNA was observed in the ribosome structure of a post-initiation complex, although the base-pairing conformation was not a typical Watson-Crick type [7]. At the hybrid state the small subunit is rotated with respect to the large subunit that is called ratcheting or ratchet-like intersubunit rotational movement [8]. It was for the first time visualized by cryo-electron microscopy (cryo-EM) and particle classification procedures that the hybrid state of both A/P and P/E tRNAs in an authentic factor-free ribosome complex during translocation [9]. Such an intersubunit rotation of pre-translocational ribosomes was observed to be spontaneous in the absence of elongation factor G (EF-G) [10]-[12]. It is also important to note that the ribosome binding the peptidyl-tRNA at the $\mathrm{P}$ site is locked, or in other words, does not rotate [13]. Accordingly, in order to return by elevating energetically from the classical P/P and E/E state to the classical $\mathrm{A} / \mathrm{A}$ and $\mathrm{P} / \mathrm{P}$ state, the step of codon recognition in participation of aa-tRNA·EF-Tu·GTP ternary complex (TC) as well as a universal mechanism of the long helix formation between small and large rRNAs, that was proposed on the basis of universally conserved nucleotide complementarity [14] would be required. Although the 3D arrangements of the three tRNAs in both classical and hybrid states have been clarified, why and how E-site tRNA is automatically expelled from the E site when A-site tRNA turns out to be cognate can be explained neither by X-ray structures nor by cryo-EM structures of the whole ribosome. Moreover, how high accuracy in translation can be achieved has never been explained in the light of the 3D structures of the whole ribosomes. It has been known that the first step of codon-anticodon recognition only expels the TC of noncognate aa-tRNAs with accuracy of about 200/10,000 [15]. The mechanism of kinetic proofreading at the step of GTP hydrolysis was proposed to expel the TC of near-cognate aa-tRNA to enhance the accuracy up to 5/10,000 [16]. It was found, however, long time ago that the accuracy is reduced by the use of high $\mathrm{Mg}^{2+} \mathrm{con}-$ centration [17]. Fig. 1 of [18] has shown that incorporation of near-cognate aa-tRNA into A site sharply in-

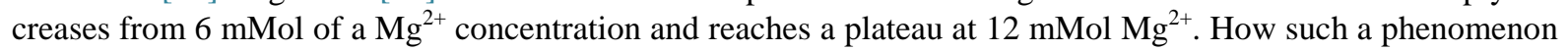
could be realized on the 3D structure of the ribosome?

\section{Universal Rule of Complementarities of rRNAs and tRNAs}

It was found that the region of nucleotides 1064 - 1077 of 16S rRNA in Esherichia coli sequence is complementary with the region of nucleotides 2653 - 2666 of 23S rRNA [14], that is called sarcine-ricin loop (SRL) (Figure 1).

The 12 nucleotide sequence of 23S rRNA, AGUACGAGAGGA, is the longest universally conserved among all known rRNAs [20]. The nucleotides at the positions 1068-1073 of 16S rRNA constitute the 5'-side of secondary structure, helix 35 of 16S rRNA (h35), while those at the positions 2653 - 2658 and 2663 - 2666 are the head region of H95. The other side of h35 contains the region of universally conserved sequence 1103-1107

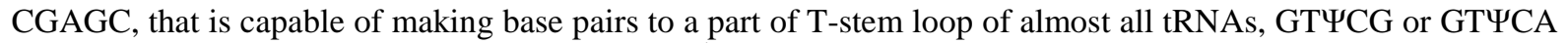
at the positions $53-57$ in the case of yeast tRNA ${ }^{\text {Phe }}$. This relationship holds for all kind of living cells including eubacteria, archaea, chloroplasts, eukarya, and mitochondria [14]. The left-most structure in Figure 1 plays an important role in GTP hydrolysis, while another structure on the second from the right-most side seems to be that of transition state. Although such a long helix has been observed by neither X-ray analysis nor cryo-EM, a model building technique can show how it looks like (Figure 2). In this structure, the possible base pairs, G2655-U1075 and G2659-C1071, are unpaired, but it is consistent with many cases in eukarya, because the 


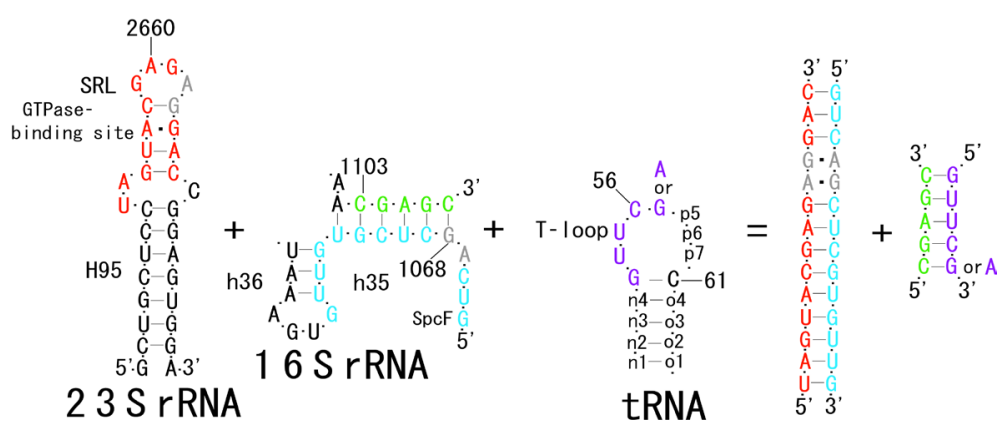

Figure 1. Universal rule of complementarities between 23S and 16S rRNAs and between 16S rRNA and tRNA [14]. SpcF denotes a spectinomycin footprint site [19].

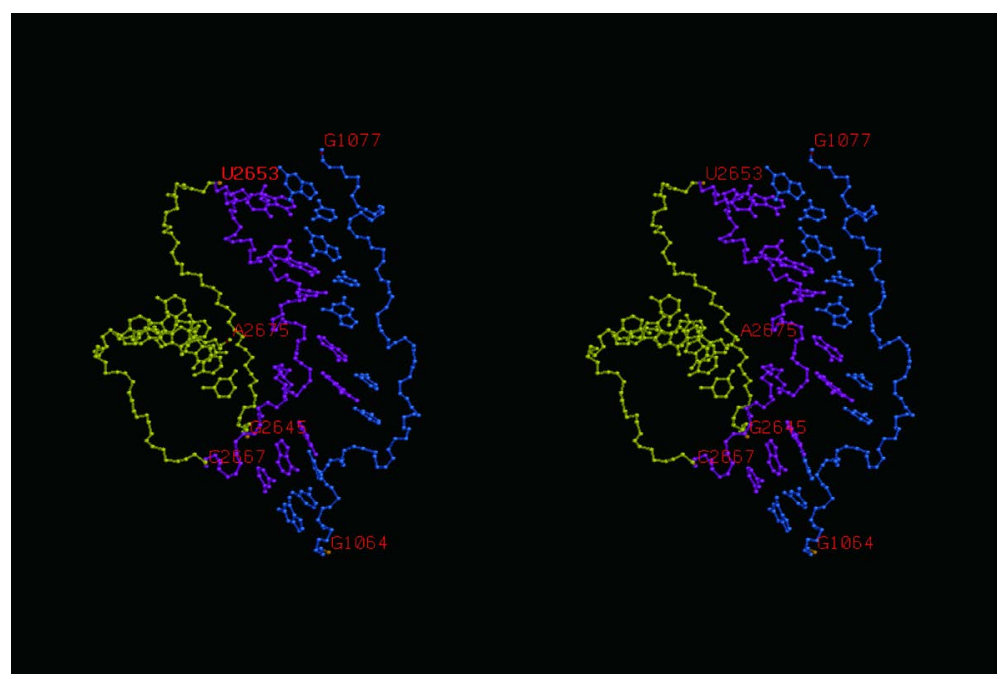

Figure 2. Stereo view of main-chain representation for double-stranded helix formed between SRL-region, U2653-C2666, of 23S rRNA, which is drawn in magenta, and the region G1064-G1077 of 16S rRNA in skyblue. The other region in yellow is G2645-C2652 and C2667-A2675 of H95. Some base pairs in H95, such as 2650-2652/2668-2670, are unfolded. Only main chains and base-pairing bases are drawn for simplicity. Red letters denote the locations of nucleotides, G1064, G1077, G2645, A2675, U2653, and C2667, respectively.

corresponding bases are guanines instead of U1075 and C1071, respectively [14]. Three base pairs in H95, C2652-G2668, C2651-G2669, and U2650-A2670 are unfolded. It is also understandable, because the first one of the three is A-C pair in some cases. Before achieving the structure shown in Figure 2, the regions of nucleotides in the 5'-side of h35 must be unfolded. This state can be obtained by the interaction of aa-tRNA in the TC of EF-Tu.GTP with the 3'-side of h35. The region of 1103-1107 of 16S rRNA, CGAGC, is able to interact with the GTYCG or GTYCA at the T-loop of aa-tRNA in the TC with EF-Tu-GTP. Such a model-building work started on the basis of atomic coordinates presented by the Protein Data Bank data bases, 2HGR and 2HGU [2]. It is also noteworthy that the region of 1064 - 1068, GUCAG, as well as the nucleotide C1192 of 16S rRNA constitute the binding site of an antibiotic spectinomycin, the conformation of which was clarified by X-ray structure (see Fig. 4 of [21]). Since it has been known that spectinomycin inhibits EF-G dependent translocation [22] [23], the conformational change of the above region of 16S rRNA must have something to do with the mechanism of translocation. Although the binding site of both EF-G. GTP complex and aa-tRNA.EF-Tu.GTP TC on the 30S subunit ribosome could be similar, a recent result of $\alpha$-sarcin-treated ribosomes has clarified that cleavage of SRL inhibited EF-G binding, GTP hydrolysis, and mRNA-tRNA translocation, while it slightly affected binding of the TC to the ribosome [24]. A big difference in binding of the GTP complexes between EF-G 
and EF-Tu·tRNA from the viewpoint of the present work is the absence of helix formation of the region 1103 1107 of 16S rRNA with the region of EF-G corresponding to the T-loop of tRNAs. Accordingly, both spectinomycin binding to the nucleotides, G1064, C1066 and C1192 of 16S rRNA [21] and splitting of the nucleotide bond between A2660 and G2661 in SRL [24] obstructs the transition from the left-most conformations to the second from the right-most conformation in Figure 1. This transition seems to produce movement of the 30S subunit relative to the 50S subunit [8]-[25]. The long helix formation represented by the second from the rightmost side in Figure 1 could be the source of driving force from the pretranslocation (PRE) state to the posttranslocation (POST) one.

\section{U33-Extended and U33-Folded Forms of the Anticodon Loop of tRNA}

Uridine 33 at the 5'-side corner of the anticodon loop of tRNA is universally conserved except for cytidine 33 in some initiator formylmethionyl-tRNAs. The uridine base of U33 is folded inside the anticodon loop of tRNAs observed so far by X-ray crystallography, and hydrogen-bonded to a phosphate oxygen atom of 36th nucleotide of the anticodon [26]. This structure is referred to as U33-folded form in this paper. This conformation of the codon-anticodon helix of U33-folded form does not seem stable enough. The U33 base can be expected to stick out or to be exposed if the conformation of the codon-anticodon helix assumes that of an ideal A-form RNA. Such a structure could be a little bit unstable because of the exposed U33 base compared to the hydrogenbonded U33 base of the U33-folded form. And yet, the stability of the U33-extended form heavily depends on the stability of the codon-anticodon helix, and seems to play an important role in enhancing the accuracy of translation. The relative stability between the U33-folded and extended forms must also depend on the concentration of $\mathrm{Mg}^{2+}$ of the buffer solution used in the experiments. If the $\mathrm{Mg}^{2+}$ concentration is pretty high, shortrange interactions would be predominant. It is said that the $\mathrm{Mg}^{2+}$ concentration inside muscle cells is about 1 mMol that is about the same as that outside the cells. On the other hand, a $\mathrm{Mg}^{2+}$ concentration used for crystallization of whole ribosomes is $10 \mathrm{mMol}$ or much higher in some cases. Crystal making people say that they use the concentration as high as required for obtaining a good crystal. The conformation of the anticodon loop of tRNAs in the living cell must be more dynamic. The difference in the positively charged ions between the living ribosomes and the ribosome crystals are mainly compensated by $\mathrm{K}^{+}$, if the environment of ribosomes are about the same as the muscle cells. A recent result of X-ray structure of the decoding site has shown that the G-U base pairs of near-cognate codon-anticodon interactions at the first position (between mRNA/Phe and tRNA ${ }_{2}^{\text {Leu }}$ ) and at the second position (between mRNA/Cys and tRNA ${ }^{\text {Tyr }}$ ) cannot be discriminated from the cognate G-C and A-U base pairs, respectively [27]. The sharp increase of the near-cognate GTPase reaction between Poly (U)programmed ribosomes and Leu 2 TCs from $6 \mathrm{mMol}$ to $12 \mathrm{mMol}$ (See Fig. 1 of [18]) strongly suggests that deprotonation of N3 and protonation of $\mathrm{O} 4$ of uridine base of $\mathrm{G}-\mathrm{U}$ pair cannot be distinguished from G-C pair [27], and that the conformational change of the anticodon loops from the U33-extended to U33-folded form of the ribosomes do not discriminate the near-cognate Leu 2 -tRNA from the cognate Phe-tRNA. Figure 3(a) shows a different 3D structure for the near-cognate G-U pair at the first position of the codon-anticodon helix on the basis of the model building technique of the present work, in which the U33 base, which is drawn in magenta, sticks out from the anticodon loop, although a $\mathrm{Mg}^{2+}$ hexahydrate still binds between OP2 atom of YG37 and O2 atom of O2' methylated OMC32. The U33-extended form of the anticodon loop would destabilize the loop structure. Besides, deprotonation from the collision of N1-H of guanine base and N3-H of uridine base resulting in the movement of the proton to the $\mathrm{O} 4$ atom of the uridine base might be preferable under the $\mathrm{Mg}^{2+} \mathrm{excess}^{2}$ conditions. Fig. 1 of [27] also shows that the P/A-kink of the mRNA is widely open as the kinks obtained by the other X-ray structures of the ribosomes [1]-[6] and supported by binding two clusters of $\mathrm{Mg}^{2+}$ hexahydrate. On the other hand, the present study can demonstrate a different type of P/A- and E/P-kinks in Figure 3(b), each of which is supported by one cluster of $\mathrm{Mg}^{2+}$ hexahydrate. The difference of the kink structures of the present work from those in the X-ray structures can be rationalized on the basis of the low $\mathrm{Mg}^{2+}$ concentration described above. In contrast to Figure 3(a), Figure 3(c) and Figure 3(d) show the structural difference in the A-site codon-anticodon helix between the cognate case in $\mathrm{C}$ and the near-cognate case in $\mathrm{D}$. In these two structures, the bases of both U33 and OMC32 as well as YG37 are exposed in order to represent a delicate difference between the cognate and near-cognate codon-anticodon helices. As a result of the difference, a $\mathrm{Mg}^{2+}$ hexahydrate between OP2 atom of YG37 and O2 atom of OMC32 is expelled, giving rise to a slight difference in the interactions between the bases of U33 and A1396. The interactions of A1492 and A1493 to the minor groove of the codon-anticodon helix can be achieved almost the same as those in the X-ray structures [27], but the interaction 
of G530 is rather difficult without a conformational change in the 530 loop due to the big conformational change in the ASL structure as well as the big shift in the location of h45, which will be described later. The near-cognate G-U base pair at the first bases of the A-site codon in Figure 3(d) is quite the same as that of Figure 3(a), but resulting in a delicate but recognizable difference from the cognate case in Figure 3(c), while the structure shown in Figure 3(a) cannot be achieved because of its big structural difference from the corresponding one in [27].

The exposed base of U33 in the U33-extended form is supposed to be supported by base-pairing with a certain universally conserved adenine base at the decoding site of 16S rRNA, the secondary structure of which is shown in Figure 4. Such base pairs are long-range interactions in contrast to the hydrogen bonded U33 base inside the anticodon loop so far observed in X-ray structures. Accordingly, a transition between U33-folded and U33-extended forms would be more frequent in the physiological condition of the $\mathrm{Mg}^{2+}$ concentration. Figure 4 shows a schematic drawing of secondary structure of 16S rRNA at the decoding site. Although the G-C pair between C1399 and G1504 has been predicted [4] [5] because of its high conservation, it was not confirmed in

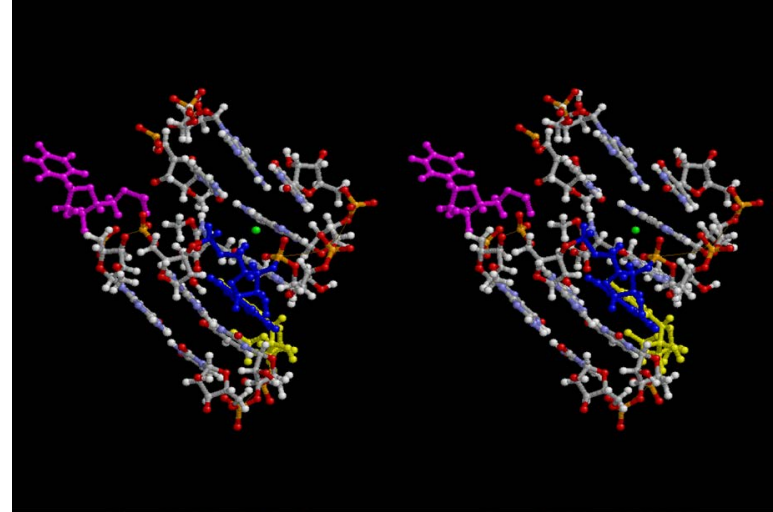

(a)

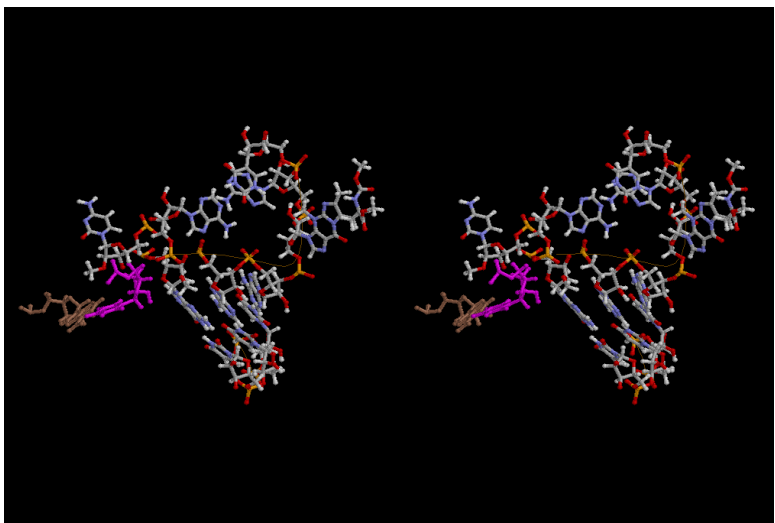

(c)

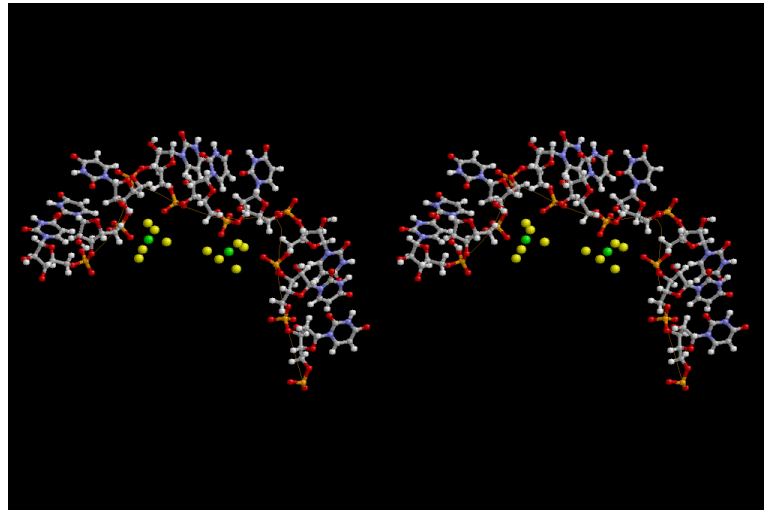

(b)

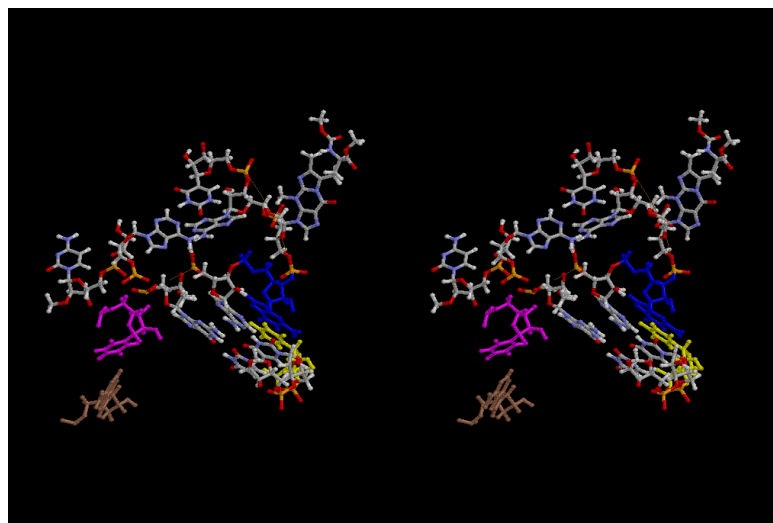

(d)

Figure 3. (a) Virtual picture of stereo view for the near-cognate G-U wobble base pair, which is drawn by blue guanine and yellow uridine, at the first position of the A-site codon-anticodon helix. $\mathrm{Mg}^{2+}$ ion in green is kept between $\mathrm{O} 2 \mathrm{P}$ atom of YG37 and $\mathrm{O} 2$ atom of 2'-O-methylated OMC32. In order to keep the wobble base pair, the base of U33 in magenta must be extended outward and destabilized because of the loss of hydrogen-bonding energy; (b) Stereo view of mRNA kinks used in the present work. Three codons at the $\mathrm{A}, \mathrm{P}$, and $\mathrm{E}$ sites, that are composed of all uridines, and two clusters $\mathrm{Mg}^{2+}$ hexahydrate binding at the P/A- and E/P-kinks, by representing green big ball for $\mathrm{Mg}^{2+}$ ions and yellow big balls for water molecules. The sharp kinks are formed by binding one $\mathrm{Mg}^{2+}$ hexahydrate at one kink instead of two, which are observed in X-ray structures; (c) Stereo view of cognate A-site codon-anticodon helix and the ASL of the present work, in which U33 of the ASL and A1396 at the decoding site of 16S rRNA are drawn in magenta and brown, respectively. Significance of A1396 will be described in the following Figure. This picture is shown so as to look similar to that in (a) as far as possible; (d) Stereo view of near-cognate A-site codon-anticodon helix and the ASL of the present work, in which G-U wobble base pair at the first base pairs of the A-site codon-anticodon helix is drawn in the same colors as those in (a) and both U33 and A1396 are as those in (c). 


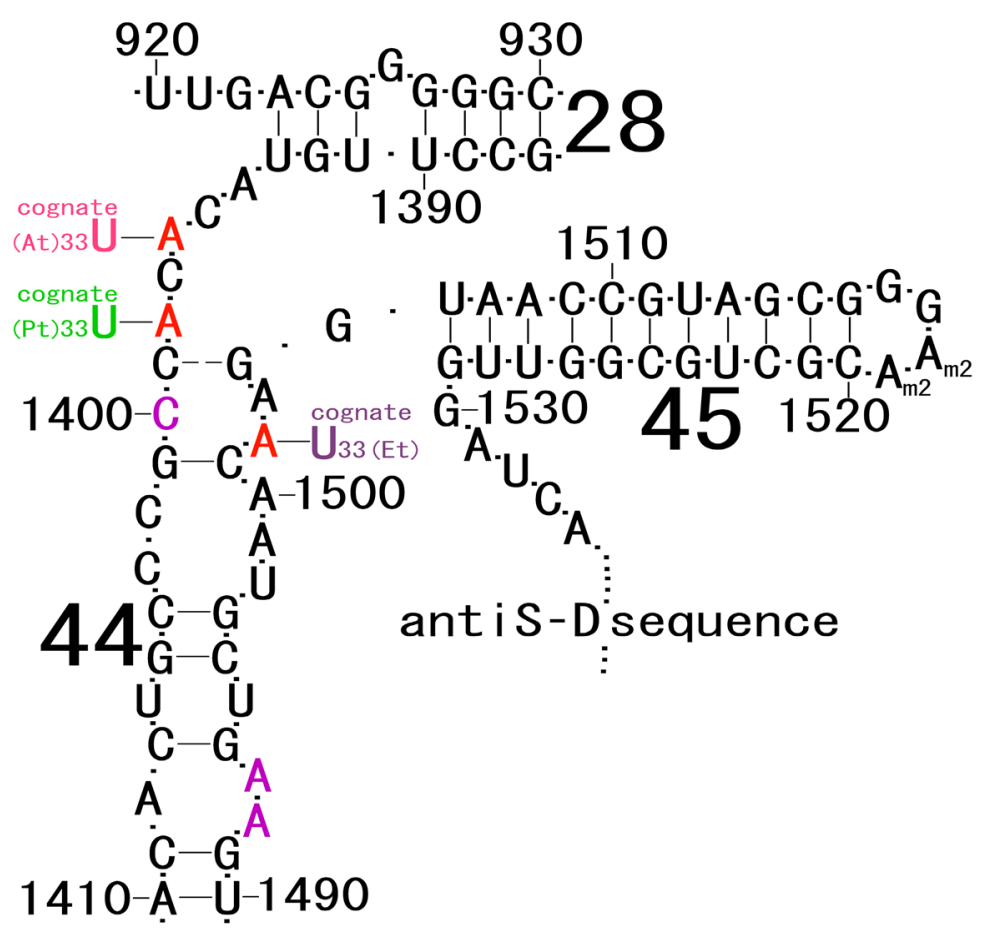

Figure 4. Assignment of three nucleotides, A1396, A1398, and A1502, on the secondary structure of the decoding site of $16 S$ rRNA. Small numerals are residue numbers of 16S rRNA [4], while large numerals denote helix numbers [5]. Base pairs are represented by single bars for both A-U and G-C pairs, while two short bars between C1399 and G1504 represents that the G-C pair is formed to reject either U33 at the A site or at the E site and not formed in the transition state in which all three U33 bases at the A, P, and E sites are base-paired with A1396, A1398, and A1502, respectively. Two $A_{m 2}$ at the positions of 1518 and 1519 are universally conserved dimethylated adenines. See text for colored nucleotides at positions, 1400, 1492 and 1493. S-D denotes Shine-Dalgarno.

the crystal structure of 2HGR [2]. On the other hand, G1401-C1501 pair as well as the base stacking of C1400 to the first base of the anticodon of P-site tRNA [28] was confirmed. It has been known that both adenines and cytidines in the decoding site are universally conserved. Accordingly, it was quite natural to think that U33 base of the U33-extended form of P-site tRNA could be base-paired with A1398. It necessarily leads to the assignment of A1396 to the base-pairing partner of the U33-extended form of aa-tRNA at the recognition mode of A site (T site). It seemed possible for either A1502 or A1503 with U33 of the U33-extended form of deacylated tRNA at the E site. The result of the present study has shown that A1502 is most favorable, while A1503 seems a little too far from the U33 of E-site tRNA.

\section{A-P and P-E tRNA Docking Pairs in the Pre- and Posttranslocation States, Respectively}

The universal rule of nucleotide sequence complementarities (Figure 1) was first noticed by a finding that oligonucleotide fragments such as TYCG or UUCG inhibited the binding of aa-tRNA at the A site [29]. It was found that such a highly conserved sequence exists on the 3'-side of h35 of 16S rRNA, and that the other side of h35 is complementary to the conserved loophead sequence of SRL in 23S rRNA [14]. In addition to the above finding, it was also found that various tRNA fragments containing D-loop inhibited aa-tRNA binding at the A site [29]. This strongly suggested that the T-loop of aa-tRNA making base-pairs with the 3'-side of h35 and the D-loop of aa-tRNA making base-pairs with the T-loop of P-site tRNA are an essential necessary requirement for the aa-tRNA entering the A site. This idea was combined with the $\alpha-\varepsilon$ model of [30], resulting in the tRNA docking pair model [31]. In contrast to the X-ray structure of the elbow region of the isolated L-form tRNA, in which G19 is paired with C56 as a Watson-Crick type, but G18 is hydrogen-bonded to $\Psi 55$ in an irregular fashion [26], the universally conserved guanines, G18 and G19, of aa-tRNA at the T site are base-paired with the other universally conserved cytidines, C56 and C61, of P-site tRNA, respectively, and also G18 and G19 of 
P-site tRNA with C56 and C61 of E-site tRNA, respectively. Such a big difference in the shape of tRNA between the isolated L-form of X-ray structure [1] [2] [26] and the docking model of the present work also comes from a big difference in the $\mathrm{Mg}^{2+}$ concentration, as mentioned above.

The results of X-ray structures of the whole ribosomes have shown that two conserved regions of 16S rRNA, the ridge containing G1338 and A1339 near h29 and the head of 790 loop, constitute barriers that block a translocational movement of P-site bound tRNA toward E site, if we assume that such a movement is just a simple-minded translation from the crystallographic $\mathrm{P}$ site to the crystallographic E site. Transition from the X-ray structure to the predicted one of the present study would start from the U33-extended form of aa-tRNA by interaction between U33 (aa-tRNA) and A1396, which is exposed on the X-ray structure, as well as the base-pairing of GTYCG (or GTYCA) of aa-tRNA with CGAGC1107. The effector region in the body of elongation factors, both EF-Tu and EF-G, would collide with the long helix composed of h30, h32, and h34 [4] [5], resulting in accomplishment of the long-range interactions between the SRL-region of 23S rRNA and the 5'-side of h35 of 16S rRNA. Under the condition of fixing the location of A76 of both P- and E-site tRNAs, the U33-extended forms make the distance between G18 (P-tRNA) and C56 (E-tRNA) shorter and C56 (P-tRNA) closer to G18 (aa-tRNA), and also, the twisting makes splitting of D-loop from T-loop for both P- and E-site tRNAs at their respective elbow regions. Such a conformational change for both $\mathrm{P}$ - and E-site tRNAs allows base-pairings between G18 (aa-tRNA) and C56 (P-tRNA) and between G18 (P-tRNA) and C56 (E-tRNA), and successively, G-C pairs between G19 (aa-tRNA) and C61 (P-tRNA) and between G19 (P-tRNA) and C61 (E-tRNA), respectively. Thus, the A-P-E tRNA docking triplet form can be completed, and finally accomplishes the codon-anticodon helix at the $\mathrm{E}$ site. This is a series of conformational changes after unfolding of h35 by the collision with the body of elongation factors, EF-Tu and EF-G, ending up with formation of the codon-anticodon helix at the E site.

Figure 5(a) shows that U33 at the P site (green) is base-paired with A1398 (red), U33 at the E site (purple) is base-paired with A1502 (pink), but U33 at the A site (magenta) is not base-paired with A1396 (brown). C1399-G1504 pair is formed and shown in gold. Figure 5(b) shows that U33 at the P site (green) is base-paired with A1398 (red), U33 at the A site (magenta) base-paired with A1396 (brown), but U33 at the E site (purple) is not base-paired with A1502 (pink). C1399-G1504 pair (gold) is formed. On the other hand, Figure 5(c) shows that all three U33 at the A, P, and E sites, (magenta, green, and purple, respectively), are base-paired with A1396 (brown), A1398 (red), and A1502 (pink), respectively, at the expense of separation of the bases of C1399 and G1504 (gold). Since both tRNAs at the P and E sites are cognate, in contrast to the case of X-ray structure, aa-tRNA at the T site would take a transition-state conformation, as shown in Figure 5(c), if it is also cognate. During fluctuation of the whole structure, both residues, C1399 and G1504, would become close enough to base-pair, as they are not so far separated. Then, A-U base pair of either A site or E site is broken. Which A-U pair is broken depends on the stability of the codon-anticodon helices at the $\mathrm{A}$ and $\mathrm{E}$ sites, since the distance between the U33 base and C1400 base, for example, would become larger in the case of less stable anticodon loop. From this reason, both noncognate (before GTP hydrolysis) and near-cognate aa-tRNA (at the step of GTP hydrolysis) are rejected, while only cognate one (after GTP hydrolysis) can enter the A site to expel the deacylated tRNA at the E site from the ribosome. This would be a reasonable explanation of the mechanism of translation concerning how high accuracy could be achieved at the decoding site.

\section{How E-Site tRNA Is Expelled Automatically When A-Site tRNA Turns out to Be Cognate}

Figure 6(a) shows a stereo view of P-site tRNA (green) surrounded by various regions of 16S and 23S rRNAs. It indicates that the SRL region of 23S rRNA (yellow) is bound together with the region of G1064-G1077 of 16S rRNA (skyblue), taking the conformation (Figure 2). It is also important to note that the region of 16S rRNA is extended from the main part of $30 \mathrm{~S}$ subunit. In order to achieve the above long-range base-pairing interactions, it is essential that the long helix composed of h30, h32 and h34 of 16S rRNA should have bends of a considerable degree around the regions between h32 and h34 as well as between h30 and h32. It necessarily causes a big deviation of the barrier, G1338 and A1339, toward the joint region of h32 and h34 from its original position, and the location of h45 is changed very much. As a result, the universally conserved dimethylated adenines, $A_{m 2} 1519$ and $A_{m 2} 1520$, at the loop of $h 45$ makes a contact with the downstream of mRNA. This result can explain why those residues are dimethylated, while the X-ray structure, 2HGR [2], cannot. A1396 approaches the U33 base of aa-tRNA, while h44 of 16S rRNA makes a contact at the inner groove of A-site codon, with un- 


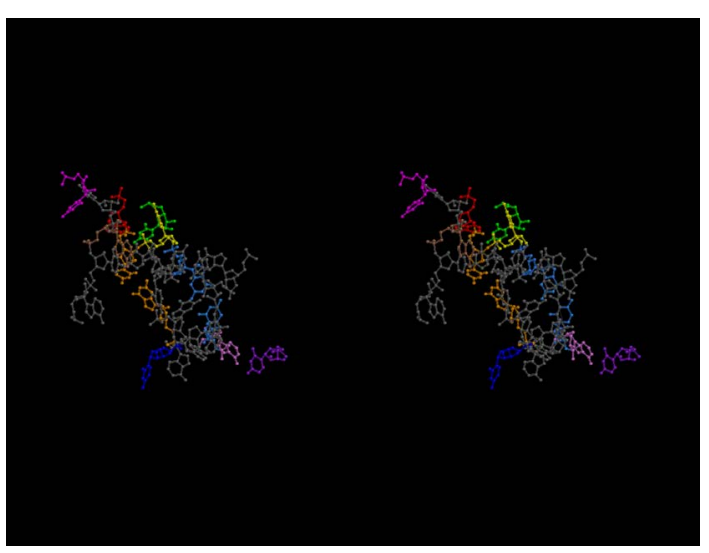

(a)

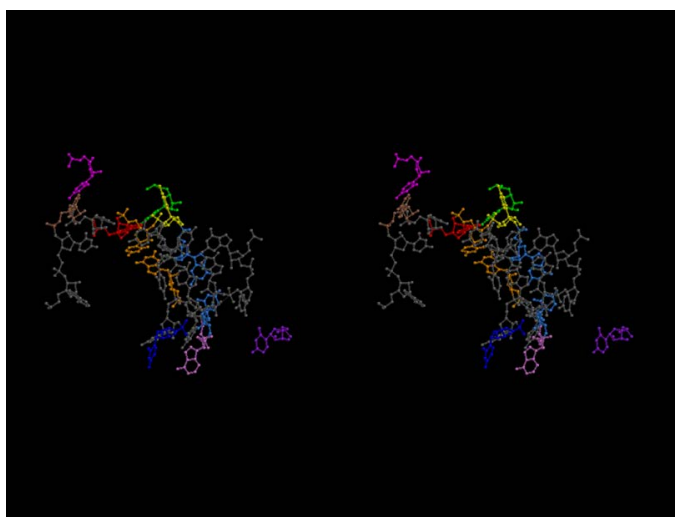

(b)

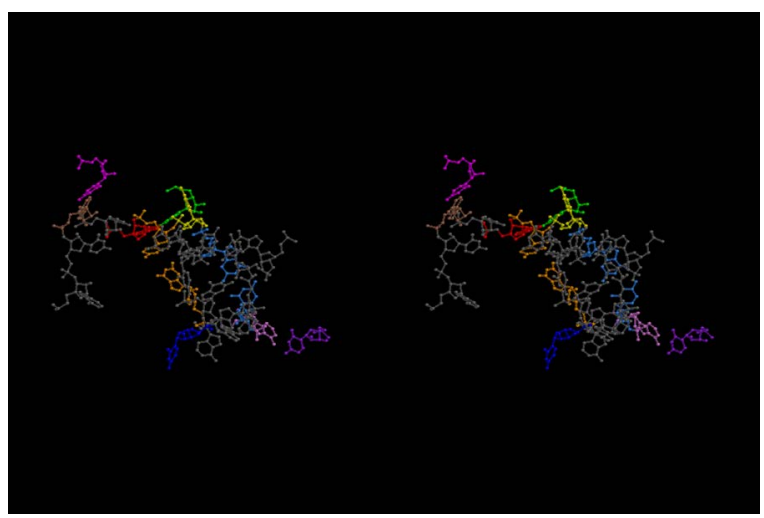

(c)

Figure 5. Stereo views of full-atom representations for the residues at the decoding site of 16S rRNA. (a) U33 of P-site tRNA (green) is base-paired with A1398 (red), that of E-site tRNA (purple) base-paired with A1502 (pink), but U33 (magenta) at the recognition mode of A site (T site) is not base-paired. A1396 (brown) is far from the U33 base (magenta). G1401-C1501 (skyblue) is always formed. C1399-G1504 (gold) is formed in this case. This structure leads to rejection of either noncognate or near-cognate aa-tRNA from the A site. C1400 is drawn in yellow, while U1506, which starts the h45, is in blue; (b) This picture is very similar to (a), but different from (a) in the following two points: U33 at the A site (magenta) is base-paired with A1396 (brown), and U33 at the E site (purple) is not base-paired with A1502 (pink); (c) This picture is also very similar to (a) and (b), except that all three U33 at the A, P, and E sites are base-paired with A1396, A1398, and A1502, respectively, but C1399-G1504 (gold) is broken.

iversally conserved A1492 and A1493 [32].

Figure 6(b) shows the transition-state conformation of three tRNAs, in which E-site tRNA as well as both Aand P-site tRNAs are usually all cognate. This structure corresponds to the local structure of the decoding site (Figure 5(c)). When U33 of the A-site tRNA is base-paired with A1396 and U33 of the E-site tRNA is not base-paired with A1502 (Figure 5(b)), the deacylated tRNA at the E site (purple) in the three tRNA binding structure changes its conformation from the U33-extended and docking with P-site tRNA state to the U33-folded and isolated form (Figure 6(c)). Here, the deacylated tRNA is just leaving the ribosome in the direction of L1 stalk. Figure 6(d) shows what would happen to the T site when the conformations of nucleotides at the decoding site are such as those in Figure 5(a). When the aa-tRNA (magenta) is noncognate, the base-pair of the codon-anticodon helix would not be formed without need of U33-A1396 base-pair formation. In the case of either near-cognate or cognate, the codon-anticodon helix could become an ideal A-form, and the help of U33-A1396 would be very important at the step of GTP hydrolysis. After fluctuation of the conformations at the decoding site between those as in Figure 5(c) and Figure 5(a), the helix at the T site would be broken, if the one at the E site is a little stronger. Then, the conformation of the aa-tRNA (magenta) would become a U33-folded form with its isolated L-form elbow region, and leaves the ribosome in the direction of L7/L12 stalk. At the instant, the conformation of the A-site codon would loosen, and become ready for accepting the next anticodon of aa-tRNA 


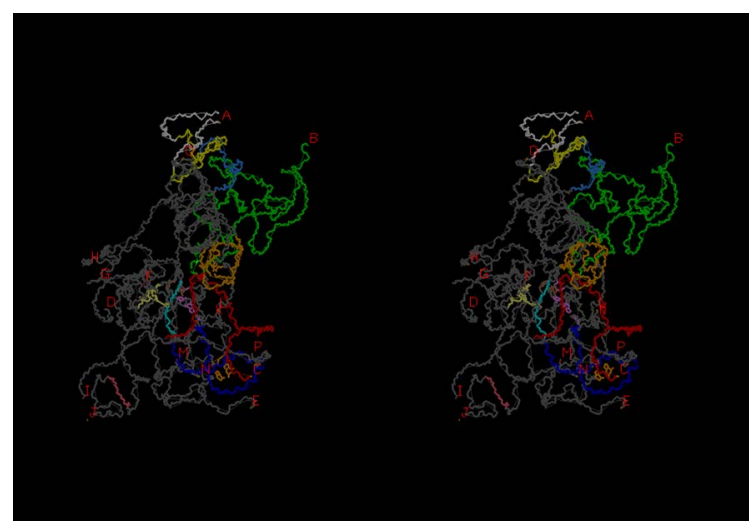

(a)

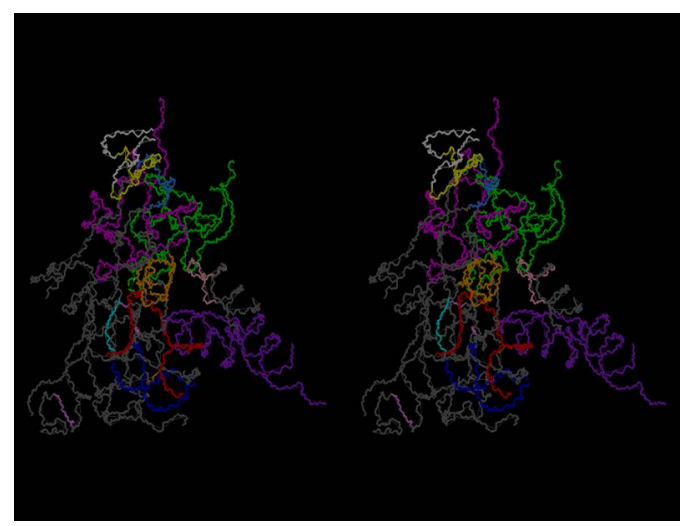

(c)

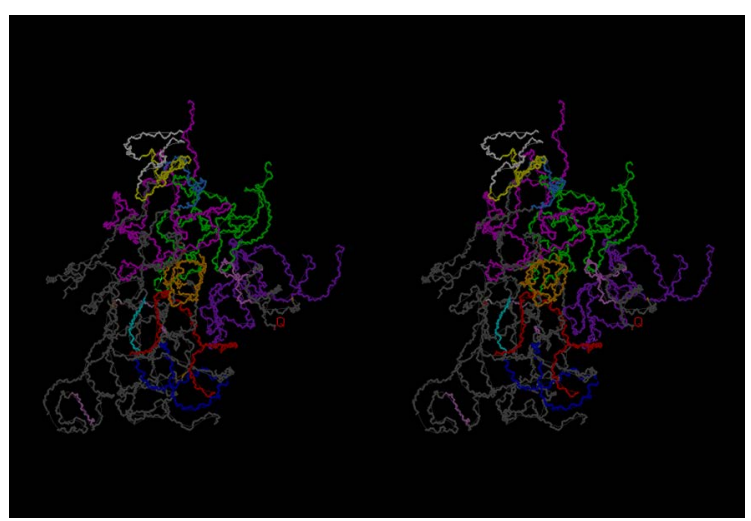

(b)

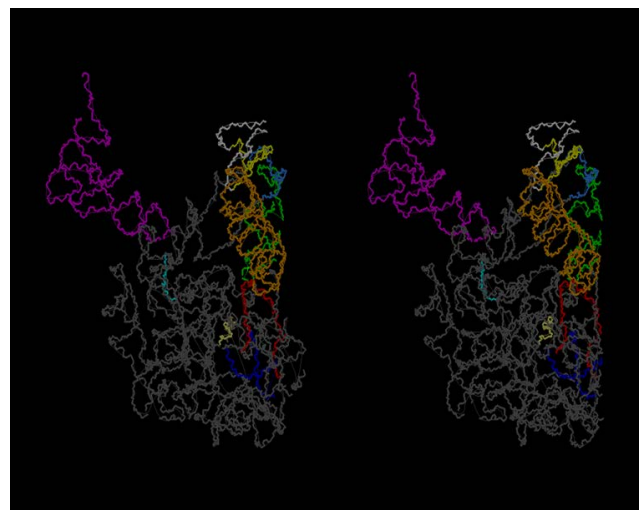

(d)

Figure 6. Stereo views of main-chain representations for the conformations of tRNAs and mRNA. (a) P-site tRNA (green) in the center. Nucleotides are G2645-C2652 (white) and G2668-A2675 (white), with a label A indicating the location of G2645. The region of U2653-C2667 (yellow) is the SRL of 23S rRNA. Label B denotes the location of A76 of tRNA at the P site. mRNA (red) with label C for its first residue. The following regions of $16 \mathrm{~S}$ rRNA are shown in different colors: G1064-G1077 (skyblue), C1103-C1107 (cyan), G527-A533 (yellow tint), U1189-A1191 (pink), G1338-A1339 with bases (gold), A1408-A1420 (orange), G1480-A1493 (orange), U1506-G1529 (blue), C1399-G1504 pair with bases (violet), and A1396 (brown). Labels from D to P denote the locations of C545, G922, U956, U991, A1041, G1117, A1180, A1236, G1334, A1350, C1384, C1452, and U1542, respectively; (b) Transition-state three tRNAs, A-site tRNA (magenta), P-site tRNA (green), and E-site tRNA (purple). This structure corresponds to the local picture of the decoding site represented in Figure 5(c). Red label Q denotes the location of C805 of 16S rRNA; (c) Three tRNA binding model, in which E-site tRNA (purple) takes an isolated L-form with its U33-folded anticodon loop. This structure corresponds to the local picture of the decoding site represented in Figure 5(b); (d) Corresponding to the local picture of the decoding site in Figure 5(a), aa-tRNA (magenta) takes an isolated L-form with its U33-folded anticodon loop.

as a TC, that would have probably been waiting for opening the A-site codon by using the 3'-side of h35 and A1394. A1394 is also highly conserved, although it is less conserved than A1396 and A1398.

\section{Translocation as a Rotational Movement of A-P tRNA Docking Pair to P-E Pair}

The effect of spectinomycin on the EF-G-dependent translocation published in the old papers [22] [23] are mentioned before. Recently, Fredrick and Noller did not observe such an effect during the course of study on sparsomycin-dependent translocation [33]. On the other hand, Peske et al. [34] have concluded that EF-G produces conformational rearrangements on the ribosome in the vicinity of the decoding region on the 30S subunit and that spectinomycin has a direct inhibitory effect on tRNA-mRNA movement, that is quite consistent with the basic assumptions of the present work. Comparison of Figure 6(b) with Figure 6(c) and Figure 6(d) suggests that translocation is not a translational movement of tRNAs from the crystallographically obtained P site to the E site but a rotational one around an axis that is close to both A- and E-site tRNAs. If the aminoacyl group remains at the A site and the P-site tRNA is still peptidylated, translocation would not occur because of locking at the P 
site [13]. Peptidyl transferase reaction results in peptidyl-tRNA at the A site and deacylated tRNA at the P site, the state of which automatically brings about $\mathrm{P}$ and $\mathrm{E}$ sites on the $50 \mathrm{~S}$ subunit. This is due to a rotational freedom around the hinge between H14 and H22 (nucleotides AA423) and a rigid binding of two helices, H22 and H88 at a pseudoknot CCAU416/AUGG2410 of 23S rRNA. C2394 and G2421 at the base of H88, which is empty as a result of the process shown in Figure 6(c), could turn to catch the adenine base of the CCA end of deacylated tRNA at the P site [3]. According to chemical modification experiments, 2'-hydroxyl groups of the nucleotides 71 and 76 of the deacylated P-site tRNA can be recognized by nucleotide C1892 in the minor groove of $\mathrm{H} 68$ and A2433 and A2434 at the base of H74 of 23S rRNA, respectively [35]. Those residues also constitute part of the E site on the 50S subunit. It would expel the P-loop at the loop of H80 as well as A2450 and its associated nucleotides of the central loop of 50S subunit from the CCA end of P-site tRNA, that is the P site on the 50S subunit, and transfer them to the CCA end of the neighboring A-site tRNA. These are what we call P/E and A/P hybrid states. Such a transition would not occur if either the 3'-end of P-site tRNA is peptidylated or some of the above key nucleotides are chemically modified. This could be an explanation as to why peptidyl-tRNA bound to the $\mathrm{P}$ site locks ribosomal functions such as translocation, termination, and recycling [13]. Figure 7(a) shows that the foot of H88 (cyan), where C2395 and C2421 are situated, reaches the adenine base of P-sitet RNA (green), while the P-loop of H80 (red) catches the bases of C74 and C75 of A-site tRNA (magenta). This is the 3Dstructural representation of the 3 '-end regions of tRNAs at the A/P and P/E hybrid states. When EF-G.GTP complex comes to occupy the space of A-site tRNA, the A-P tRNA docking pair rotates around the axis passing closer to the A-, P- and E-site tRNAs to reach the site of P-E tRNA docking pair at the classical P/P and E/E states. The 3D structural representation of them can be shown as in Figure 7(b).

\section{How Elongation Factors Work on the Present Models to Produce GTP Hydrolysis?}

The recent result of the crystal structure of the ribosome bound to EF-Tu and aa-tRNA [36] does not seem to fully explain the mechanism of accommodation of aa-tRNA into A site. Starting from the tRNA structure in magenta in Figure 6(d), which is either leaving the ribosome or entering the ribosome as a TC with EF-

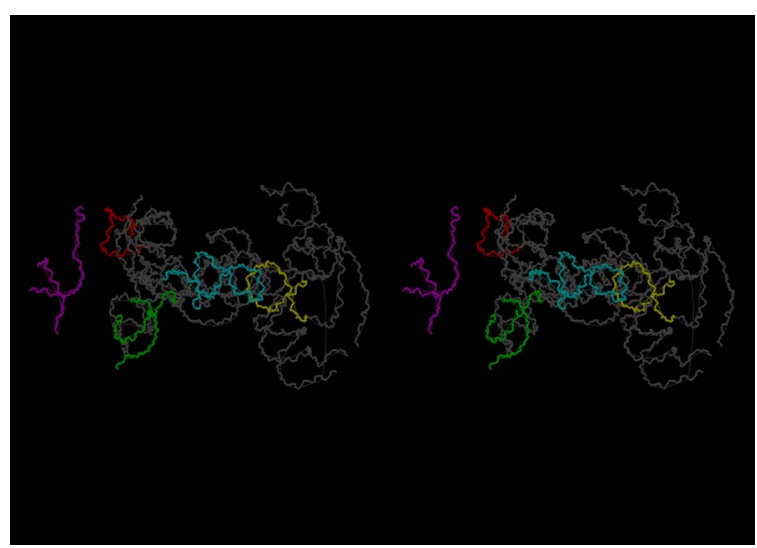

(a)

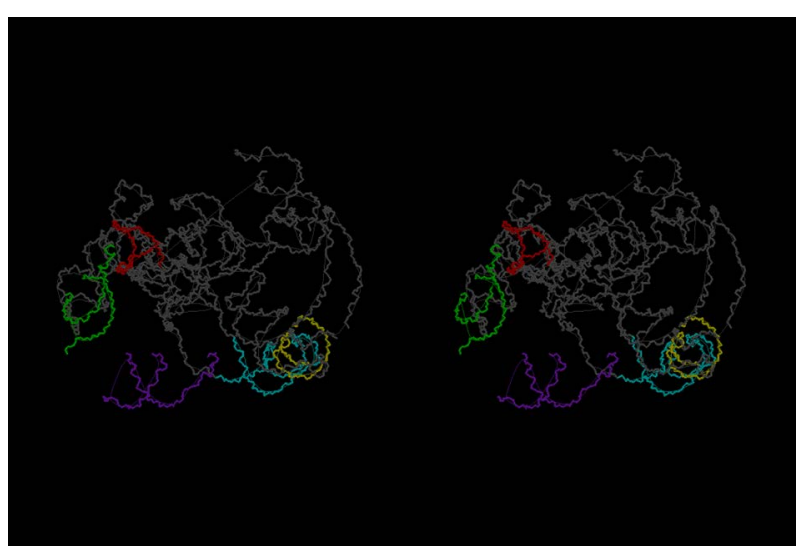

(b)

Figure 7. Stereo views of main-chain representations for the conformations of the 3'-end regions of tRNAs surrounded by the nucleotides at the H80 (P-loop) and H88. It is known that C2422 can bind to the 3'-end adenine base (A76) of deacylated tRNA at either P or E site. The loop of H88, A2407-G2410, is tightly bound with C413-U416 at the loop of H22, which is drawn in yellow, and works as widely movable long rod, by fixing its one end at the position of G424 on the 50S subunit, between the 3'-end region of P-site tRNA (green) in the PRE state, and that of E-site tRNA (purple) in the POST state. (a) The structure of 3'-end regions of two tRNAs on the 50S subunit in the PRE state. The regions of G1-U8 and A66-A76 of A-site tRNA (magenta), G1-U8 and A66-A76 of P-site tRNA (green), G2246-C2258 (H80) (red), C2395-G2421 (H88) (cyan), G406-C421 (H22) (yellow) of 23S rRNA and the other associated regions (grayish white) are drawn. This corresponds to the intermediate structure in the A/P and P/E hybrid states; (b) The structure of 3'-end regions of two tRNAs on the 50 S subunit in the POST state. The regions of G1-U8 and A66-A76 of P-site tRNA (green), G1-U8 and A66-A76 of E-site tRNA (purple), G2246-C2258 (H80) (red), C2395-G2421 (H88) (cyan), G406-C421 (H22) (yellow) of 23S rRNA, and the other regions (grayish white) are drawn. This corresponds to the structure in the classical P/P and E/E states. 
Tu'GTP, its 3' end region was modified by transplanting 3' end region of Trp-tRNA ${ }^{\text {TRP }}$ along with EF-Tu and GDP bound to a cognate codon on the 70S ribosome 2Y18 [37]. GDP structure was changed to GTP by adding $\mathrm{P} \gamma$ phosphate. The conformation of EF-Tu was changed so that the $\mathrm{P} \gamma$ atom comes closer to N6 atom of A2660 and $\mathrm{P} \beta$ atom closer to $\mathrm{N} 4$ atom of $\mathrm{C} 2658$. $\mathrm{P} \gamma$ and $\mathrm{P} \beta$ atoms are covered by the region of amino acid residues 20 26 (VDHGKTT) in EF-Tu. The G base of GTP moiety is hydrogen bonded to U2656, while the O3' and O2' atoms on its ribose ring are hydrogen bonded to N6 atom of A2657 and O6 atom of G1074 of 16S rRNA, respectively. Such a local structure of GTP binding to the present model at the interface of RSL of 23S rRNA and the 5'-half of h35 of 16S rRNA on the ribosome structure is shown in Figure 8(a). Inspection of this local structure suggests that GTP hydrolysis could occur when the region of EF-Tu along with GTP molecule moves

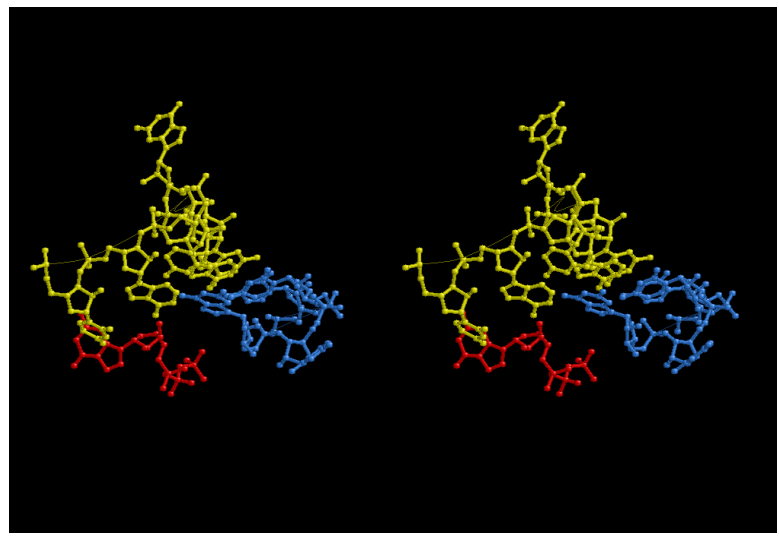

(a)

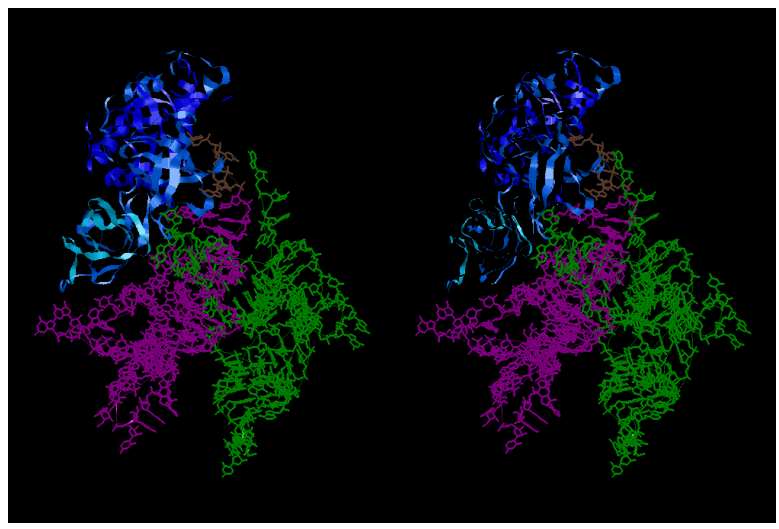

(c)

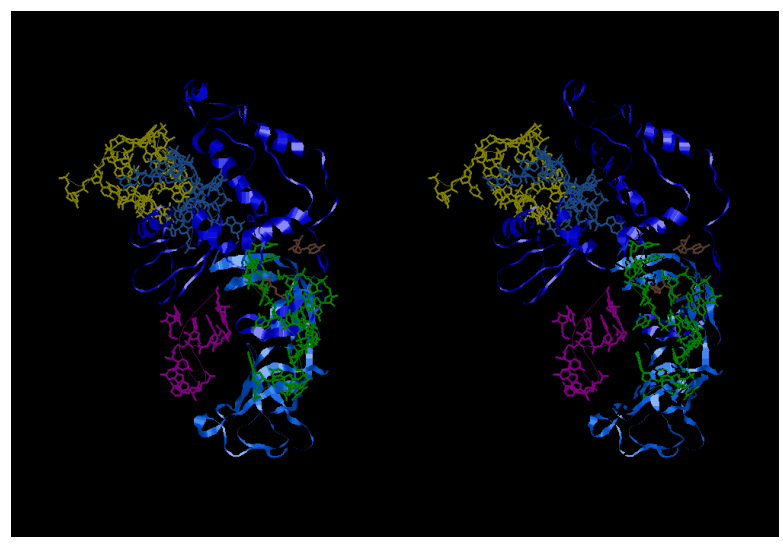

(b)

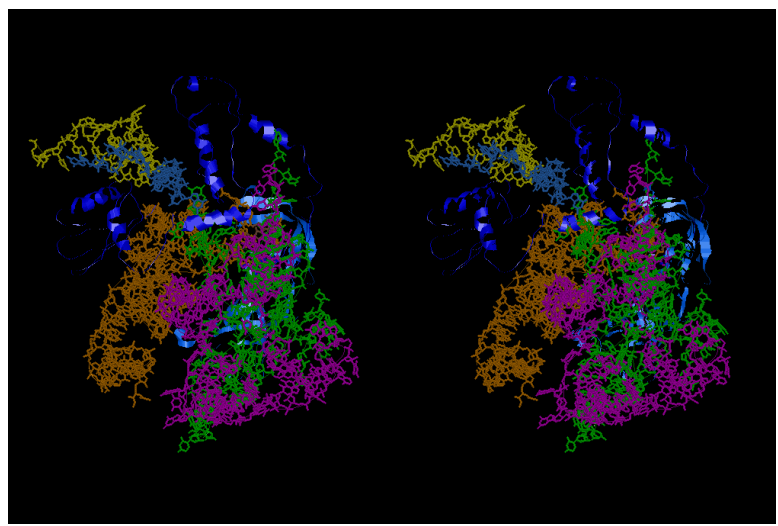

(d)

Figure 8. Stereo views of elongation factors during the course of A-site accommodation and translocation with GTP hydrolysis. (a) Stereo views of main-chain representations of GTP molecule (red) at the border of RSL from U2656 to G2661 (yellow) and 5' half of h35 of 16S rRNA from G1069 to G1072 (skyblue); (b) The same model in (a) is shown from the other direction that is from the 3' ends of P-site tRNA (green) toward the mRNA. When the codon-anticodon interaction is that of cognate, the conformational changes at the ASL and the elbow region of T-site tRNA (magenta) induces the GTP hydrolysis, ending at the conformation of A-site tRNA as shown in Figure 6(b)-(c). Blue ribbon, and the other nucleotide chains represent the peptide chain of EF-Tu from A1 to E405 with VDHGKTT (20-26) missing, RSL region (C2652-C2667 in yellow), 5' side of 16S rRNA (G1064-G1077 in skyblue), T-site tRNA (A5-U8, A14-U15, 5MC49, U68, U72 and A76 in magenta), and P-site tRNA (G45-C56 and C61-G65 in green), respectively. The regions of T- and P-site tRNAs are only shown for those in close contact with EF-Tu. The region of U72-A76 in Trp-tRNA ${ }^{\text {TRP }}$ (brown) is taken to connect the main body of T-site tRNA and the EF-Tu polypeptide regions (ribbon) stretching toward GTP binding site by A1-R190 and P-site tRNA by G191-E405; (c) The location and conformation of EF-Tu is shown after the accommodation of A-site tRNA is finished. EF-Tu leaves the ribosome immediately. The rest of the model is the same as that of Figure 6(c); (d) The ancestral form of EF-G bound to the PRE state of ribosome is shown. The body of tRNA in gold corresponds to the domain IV of EF-G. The rest of the ancestral EF-G is the same as that of EF-Tu, which appeared in (b). Since A-site is occupied by a big body of tRNA, the location of GTP binding to the border of RSL of 23S rRNA and 5' half of h35 is shifted by as far as $27 \AA$ from the $\mathrm{P}$-site tRNA. 
in the direction from N4 atom of C2658 to N6 atom of A2660 in contrast to the movement vertical to the above. When the distance between the N4 and the N6 atoms becomes larger accompanying the $\mathrm{P} \beta$ and $\mathrm{P} \gamma$ atoms, respectively, NH2 group of the N2 position of G2659 attacks the $\mathrm{P} \beta-\mathrm{P} \gamma$ bond. The total structure of EF-Tu with the related regions of T- and P-site tRNAs is shown in Figure 8(b). After GTP hydrolysis in the structure shown in Figure 8(b), the conformation of EF-Tu returns to the original one observed in the crystal structure of the ribosome bound to EF-Tu and Trp-tRNA ${ }^{\text {TRP }} 2$ Y18 [37]. Such a structure is shown in Figure 8(c). The EF-Tu molecule would leave the ribosome soon under the normal condition of low $\mathrm{Mg}^{2+}$ concentration. The conformation of the A-site tRNA is the same as those shown in Figure 6(b)-(c). Since the binding of A-site tRNA very close to P-site tRNA, the location of which is almost the same as the other structures shown in Figure 6, the cavity between the RSL region and P-site tRNA becomes a little wider, where EF-G is supposed to bind. The structure of tRNA in gold, as shown in Figure 8(d), is a putative ancestral form of EF-G, in which the tRNA-like body in gold represents the domain IV of EF-G [38], while the other part represents the rest of the whole structure of EF-Tu. If the conformational change in the part of EF-Tu occurs after GTP hydrolysis, the structure very much like the one shown in Figure 8(b) would be obtained. Because of the exposed form of the domain IV of EF-G, or the tRNA moiety in gold, would be very unstable, the structure would become immediately the same as that in Figure 8(c) after translocation. This is exactly what happened in the crystal structure of the ribosome shortly after binding of EF-G [39]. The reason why EF-G dependent translocation is inhibited by binding of spectinomycin ([22] [23] [33]) can be explained very clearly as the result of missing the contribution from the 5' half of h35 in the mechanism of GTP hydrolysis shown in Figure 8(a). In contrast to the EF-G dependent translocation, the A site tRNA accommodation catalyzed by EF-Tu does not seem to be affected by spectinomycin. The reason for this would be that the region of CGAGC1107 on the 3' half of h35 is unfolded by interaction with the GT $\Psi C G$ region of T-loop of aa-tRNA, or could be due to a slight difference in the distance between P $\gamma$ atom and 3' end of P-site tRNA (27 А).

\section{How the First Base of Stop Signals, U, Can Be Recognized?}

After translocation, the new A-site codon is open for the next aa-tRNA. Before going to further recognition of aa-tRNA, how is it judged to be either a sense codon or a stop signal such as UAA, UAG or UGA? Three kinds of release factors are known to recognize stop signals as follows: RF1 in prokaryote has the conserved tripeptide motif Pro-Ala-Thr for UAG and UAA, RF2 in prokaryote has the motif Ser-Pro-Phe for UGA and UAA, and a single eukaryotic one eRF1 has the tetrapeptide motif Asn-Ile-Lys-Ser for UAG, UGA, and UAA [40]. It was insisted that a tripeptide Pro-Ala-Thr in prokaryotic RF1 can recognize the stop signals specifically [41]. Furthermore, the above three motifs of class-1 RFs have been believed to recognize stop signals directly as if they are anticodons, and are confirmed for RF2 by cryo-EM [42] [43] and for eRF1 by analogy from its tRNA-like shape of the X-ray structure [44]. However, a big question still remains unsolved as to how the first base of stop signals, U, is recognized [40]. Here, a hypothetical mechanism is presented of base-pairing of stop signals with a conserved nucleotide sequence UUA1092 at the loop of h37. If the codon is UGG, the UGG/UUA base pairs would not be stable enough because of two consecutive wobble base pairs. It would be more favorable for such a transient helix UUA/stop signal to allow one of the tri- or tetrapeptide motifs to come close enough, and to withdraw immediately after one of the release factors achieves a direct interaction with the stop signal. It seems to be a common mechanism to all living kingdoms except for mitochondria of some protista (Trypanosoma brucei and Plasmodium falciparum) and animalia [14] because of missing h37 in their nucleotide sequence of small subunit rRNAs. UGA is used as the codon for Trp-tRNA ${ }^{\text {Trp }}$ of animalia mitochondria (See Table 12 of [45]). The evolution of tRNA ${ }^{\text {Trp }}$ seems to have been developing in relation with that of stop signals and also release factors. Figure 9 shows that the UUA sequence at the loophead of h37 (pink) is situated at a good location of finding one of the stop signals in the A-site codon (red) before any release factor comes to form a more stable complex.

\section{Negative Cooperativity between A and E Sites}

Nierhaus and his coworkers have insisted that a deacylated tRNA bound to E site and cognate to the E-site codon allosterically and negatively regulates affinity for a cognate tRNA entering to A site [46] [47]. Since any proofreading mechanism in translation other than kinetic one [16] has never been proposed, the present study has started to provide a theoretical background for the attractive proposal [46]. The present mechanism of transi- 


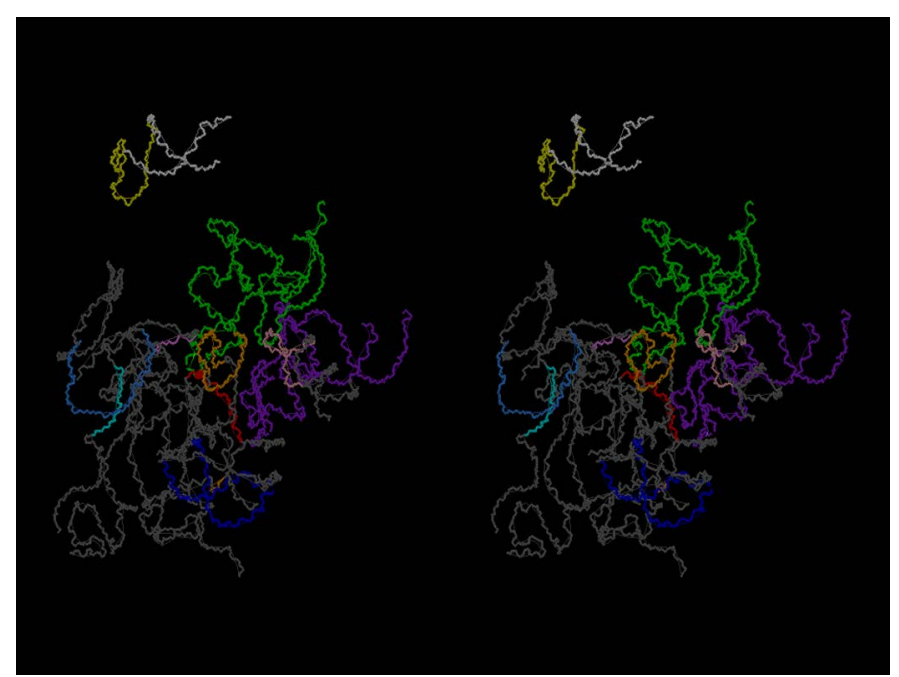

Figure 9. Stereo views of main-chain representations for the structures of the regions changed from those drawn in Figure 6(c) shortly after translocation. The base-pairing interaction between the SRL region and the 5'-end region of h35 disappears. The hypothetical interaction between the region of UUA1902 (pink) of h37 and a stop signal UAA (red) is shown. Only three codons for A-, P-, and E-site anticodons are drawn in red. One reason for the hypothesis is that h37 is missing for animalia mitochondria accompanied with a change in UGA codon usage for tRNA ${ }^{\mathrm{TRP}}$. The other regions are as in Figure 6(a) and Figure 6(b).

tion-state three-tRNA structure represents another aspect of kinetic proofreading mechanism [16], because it is accompanied by a step of GTP hydrolysis. In the present model-building works, the three-tRNA binding model at the transition state (Figure 6(b)) strongly supports the negative cooperativity between A and E sites, because A- and E-site tRNAs are very close to each other, although the two tRNAs do not have direct contacts with each other. However, the experimental findings of Nierhaus and his coworkers [46]-[50] described above have recently been refused to accept by many research groups [51]-[54]. Chen et al. [53] analysed how E-site tRNA is dissociated early in protein synthesis. They denoted the two options as the 2-3-2 tRNA and 2-1-2 tRNA pathways, where the integers indicate the number of tRNAs occupying the ribosome during the conversion of the POST complex to the PRE complex. On the 2-1-2 pathway E-site tRNA leaves the ribosome before aa-tRNA is accommodated with the A site. In this study, the model of the 2-3-2 pathway was presented, while the conformation of the tRNA anticodon loop of the 2-1-2 pathway would be the U33-folded form, and both the codon-anticodon base-pairings at the E site and the docking G-C pairings between P- and E-site tRNAs would be lost. Since the P-site tRNA slippage in the analysis of +1 programmed ribosomal frameshifting (PRF) [55] would mean a considerable degree of dynamic behavior of the anticodon loops at the decoding site, the competition of U33-extended and U33-folded forms would also be a realistic problem depending on various factors, such as $\mathrm{Mg}^{2+}$ concentration, conformation of the nascent polypeptide, specific nucleotide sequence of mRNA, etc. The transition from the U33-extended form to the U33-folded form and vice versa at the P-site tRNA under the condition that the codon-anticodon helix is kept at the E site and that the A-site codon is open would cause a P-site tRNA slippage even if the +1 frame is near-cognate [56]. In the absence of the codon-anticodon interactions at the E site, -1 PRF could also occur in a comparable probability with that of +1 PRF. If the transition gives rise to a noncognate $+1 \mathrm{PRF}$, it would soon come back to the original zero frame. Even if such a transition is rather frequent, it does not show any detectable change at the decoding site, if the frameshifting always returns to the original frame. Accordingly, the findings so far obtained about the PRFs are quite consistent with the 3D structural models presented in this study. $\mathrm{A} \mathrm{Mg}^{2+}$ hexahydrate $\left(\mathrm{Mg}\left(\mathrm{H}_{2} \mathrm{O}\right)_{6}{ }^{2+}\right)$ was found to bind to a phosphate oxygen of 37th nucleotide of the anticodon loop of yeast phenylalanine tRNA (6tna) [26]. It is located between $\mathrm{O} 2$ atom of O-methyl cytosine at the 32nd position (OMC32) and O3' atom of 36th nucleotide of the anticodon loop. This would be a result of hydrogen bonding between O4 atom of U33 and OP2 atom of the 36th nucleotide. However, if the $\mathrm{Mg}^{2+}$ concentration is getting higher and higher, U33-folded form would become prevailing. The distance between two phosphoric oxygen atoms of a kink of mRNA between the U33-folded forms of two adjacent codon-anticodon helices would be fixed around the range near $8 \AA$ by intervention of a $\mathrm{Mg}^{2+}$ hydrate. This would make the anticodon of the E-site tRNA unable to form a helix with the E-site codon. 
Nierhaus and his coworkers have been using $4.5 \mathrm{mMol} \mathrm{Mg}{ }^{2+}$ and some amount of polyamines as a buffer of physiological condition [57]. Petropoulos and Green [54] used the same buffer, and yet refused the negative cooperativity between A- and E sites [46]. Chen et al. [53] observed that the 2-3-2 pathway only occurred shortly after initiation and followed by consecutive 2-1-2 pathways and that the translation pathway is mainly determined by the PRE conformational states (either classical or hybrid). They used TAM ${ }^{15}$ buffer, which contains $15 \mathrm{mMol} \mathrm{MgAc}_{2}$ [53]. It seems to me that they are using too high $\mathrm{Mg}^{2+}$ concentration, even if they lowered it from 15 to $7 \mathrm{mMol}$ [53]. Every group should discuss with each other in terms of accuracy in translation. I insist that the 2-3-2 pathway would prevail if the PRE states are those of U33-extended and A-P tRNA docking pair with a much less $\mathrm{Mg}^{2+}$ concentration. G18-G19 as well as C56 and C61 in tRNA sequence are highly conserved for all living kindoms [14]. Proofreading mechanism in translation should be explored under a condition of $1 \mathrm{mMol} \mathrm{Mg}{ }^{2+}$ concentration.

\section{Conclusion}

The mechanism of translation was described in detail in the preceding sections. Several important points, however, still remain untouched, and should be concluded here. The point 1: The locations of tRNAs for A, P, and E sites on the X-ray structures of the whole ribosomes were distantly separated from each other, and barriers seemed to bother smooth translocation from P- to E-sites, if the translocation could occur as a simple-minded translational movement [1] [2] [6] [7]. In translocation, A-site tRNA must jump into P site, cooperatively with the previous P-site tRNA jumping into E site. It is hard to explain mechanistically, but, more difficult is to imagine how E-site tRNA is automatically expelled when a new tRNA at the A site turns out cognate. The present paper described such a mechanism in detail. Here, I can only explain very easily how X-ray structures of the whole ribosome can be obtained from my models of A-, P- and E-site tRNAs. When the $\mathrm{Mg}^{2+}$ concentration exceeds $6 \mathrm{mMol}$, the local conformations of the above three tRNAs at the anticodon loops, and their elbow regions change drastically and break the G-C pairs made between two neighboring tRNAs. In order to understand rationally the process of translocation in the X-ray structure, we must shift to our models of translocation, carry out the rotational movement of translocation, and again return to the posttranslocation X-ray structure. The formation of the long helix made by the SRL region of 23S rRNA and 5' side of h35 of 16S rRNA, which is referred to as GTPase-binding site, could only be achieved under the low $\mathrm{Mg}^{2+}$ concentration, and would disappear under the condition of crystallization of X-ray structure probably because of less flexibility of rRNAs. The point 2: Another point to be subjugated was deprotonation from N3 and protonation of O4 in the uridine base of wobble base pair under the condition of $\mathrm{Mg}^{2+}$ concentration higher than $6 \mathrm{mMol}$ making it impossible to discriminate between G-C and G-U pairs, in other words, between cognate and near cognate codon-anticodon interactions [27]. It would be essential to adopt a low $\mathrm{Mg}^{2+}$ concentration to avoid the problem, and to have a possible interaction of U33-extended form of the anticodon loop of tRNAs with the very highly conserved nucleotide sequences at the decoding site. The point 3: The mechanical movement of translocation was interpreted as a rotational movement, while hybrid states such as $\mathrm{A} / \mathrm{P}$ and $\mathrm{P} / \mathrm{E}$ were able to be achieved without need of surmounting a high energy barrier as a rotational movement of a long rod of helical rRNAs made by a rigid binding of $\mathrm{H} 22$ and $\mathrm{H} 88$ under a condition that P-site tRNA is deacylated. The point 4: GTP hydrolysis results in a big conformational change in EF-Tu with a right hand (amino acid sequence from Ala1 to Arg190) pushing the GTPase-binding site and with a left hand (from Gly191 to Glu405) pulling the whole body of P-site tRNA. The final shape of A-site tRNA is just like a sitting-up of the T-site tRNA. On the other hand, GTP hydrolysis in translocation by means of EF-G produces a little different result from that of EF-Tu. This could be due to a slight difference in amino acid sequence between EF-Tu and EF-G (one amino acid insertion) and result in a different rotational movement with EF-G's left hand pushing the whole body of A-site tRNA toward P-site tRNA and with its right hand pushing the GTP-ase binding site to break the long helix made by the SRL region of 23S rRNA and 5'-side of h35 of 16S rRNA. The big distortion introduced by formation of the GTPase-binding site is cancelled and returns to the original state. This movement of the whole ribosome represents exactly where the driving force of the rachet-like movement of the ribosome comes from.

\section{Methods}

\section{Programs and Atomic Coordinates}

The programs for manipulating 3D models in the present work were written in FORTRAN95 by the present au- 
thor. The atomic coordinates of the original forms of tRNAs and rRNAs were obtained from the Protein Data Bank. The predicted model for the virtual case of near-cognate codon-anticodon helix with high $\mathrm{Mg}^{2+}$ concentration (Figure 3(a)) was built by use of atomic coordinates 6tna [26]. In order to draw colored stereo pictures, a program called RasMol [58] was used.

\section{References}

[1] Yusupov, M.M., et al. (2001) Crystal Structure of the Ribosome at 5.5 Å Resolution. Science, 292, 883-896. http://dx.doi.org/10.1126/science.1060089

[2] Korosterev, A., Trakhanov, S., Laurberg, M. and Noller, H.F. (2006) Crystal Structure of a 70S Ribosome-tRNA Complex Reveals Functional Interactions and Rearrangements. Cell, 126, 1065-1077. http://dx.doi.org/10.1016/j.cell.2006.08.032

[3] Sergiev, P.V., et al. (2005) Function of the Ribosomal E-Site: A Mutagenesis Study. Nucleic Acids Research, 33, 6048-6056. http://dx.doi.org/10.1093/nar/gki910

[4] Gutell, R.R. (1993) The Simplicity behind the Elucidation of Complex Structure in Ribosomal RNA. In: Nierhaus, K.H., Franceschi, F., Subramanian, A.R., Erdmann, V.A. and Wittmann-Liebold, B., Eds., The Translational Apparatus, Structure, Function, Regulation, Evolution, Plenum Press, New York, 477-488.

[5] Brimacombe, R. (1995) The Structure of Ribosomal RNA: A Three-Dimensional Jigsaw Puzzle. European Journal of Biochemistry, 230, 365-383. http://dx.doi.org/10.1111/j.1432-1033.1995.0365h.x

[6] Selmer, M., et al. (2006) Structure of the 70S Ribosome Complexed with mRNA and tRNA. Science, 313, $1935-1942$. http://dx.doi.org/10.1126/science.1131127

[7] Yusupova, G., Jenner, L., Rees, B., Moras, D. and Yusupov, M. (2006) Structural Basis for Messenger RNA Movement on the Ribosome. Nature, 444, 391-394. http://dx.doi.org/10.1038/nature05281

[8] Frank, J. and Agrawal, R.K. (2000) A Ratchet-Like Intersubunit Reorganization of the Ribosome during Translocation. Nature, 406, 318-322. http://dx.doi.org/10.1038/35018597

[9] Agirrezabala, X., Lei, J., Brunelle, J.L., Ortiz-Meoz, R.F., Green, R. and Frank, J. (2008) Visualization of the Hybrid State of tRNA Binding Promoted by Spontaneous Ratcheting of the Ribosome. Molecular Cell, 32, 190-197. http://dx.doi.org/10.1016/j.molcel.2008.10.001

[10] Kim, H.D., Puglisi, J. and Chu, S. (2007) Fluctuations of tRNAs between Classical and Hybrid States. Biophysical Journal, 104, 13661-13665.

[11] Munro, J.B., Altman, R.B., O’Connor, N. and Blanchard, S.C. (2007) Identification of Two Distinct Hybrid State Intermediates on the Ribosome. Molecular Cell, 25, 505-517. http://dx.doi.org/10.1016/j.molcel.2007.01.022

[12] Cornish, P.V., Ermolenko, D.N., Noller, H.F. and Ha, T. (2008) Spontaneous Intersubunit Rotation in Single Ribosomes. Molecular Cell, 30, 578-588. http://dx.doi.org/10.1016/j.molcel.2008.05.004

[13] Valle, M., Zavialov, A., Sengupta, J., Rawat, U., Ehrenberg, M. and Frank, J. (2003) Locking and Unlocking of Ribosomal Motions. Cell, 114, 123-134. http://dx.doi.org/10.1016/S0092-8674(03)00476-8

[14] Nagano, K. and Nagano, N. (2007) Mechanism of Translation Based on Intersubunit Complementarities of Ribosomal RNAs and tRNAs. Journal of Theoretical Biology, 245, 644-668. http://dx.doi.org/10.1016/j.jtbi.2006.11.004

[15] Lotfield, R.B. and Vanderjagt, D. (1972) The Frequency of Errors in Protein Biosynthesis. Biochemical Journal, 128, 1353-1356.

[16] Hopfield, J.J. (1974) Kinetic Proofreading: A New Mechanism for Reducing Errors in Biosynthetic Processes Requiring High Specificity. Proceedings of the National Academy of Sciences of the United States of America, 71, 4135-4139. http://dx.doi.org/10.1073/pnas.71.10.4135

[17] Szer, W. and Ochoa, S. (1964) Complexing Ability and Coding Properties of Synthetic Polynucleotides. Journal of Molecular Biology, 8, 823-834. http://dx.doi.org/10.1016/S0022-2836(64)80163-7

[18] Thompson, R.C., Dix, D.B., Gerson, R.B. and Karim, A.M. (1981) Effect of $\mathrm{Mg}^{2+}$ Concentration, Polyamines, Streptomycin, and Mutations in Ribosomal Proteins on the Accuracy of the Two-Step Selection of Aminoacyl-tRNAs in Protein Biosynthesis. Journal of Biological Chemistry, 256, 6676-6681.

[19] Moazed, D. and Noller, H.F. (1974) Interaction of Antibiotics with Functional Sites in 16S Ribosomal RNA. Nature, 327, 389-394. http://dx.doi.org/10.1038/327389a0

[20] Egebjerg, J., Larsen, N. and Garrett, R.A. (1990) Structural Map of 23S rRNA. In: Hill, E.E., Dahlberg, A., Garrett, R.A., Moore, P.B., Schlesinger, D. and Warner, J.R., Eds., The Ribosome Structure, Function and Evolution, American Society for Microbiology, Washington DC, 168-179.

[21] Carter, A.P., Clemons, W.M., Brodersen, D.E., Morgan-Warren, R.J., Wimberly, B.T. and Ramakrishnan, V. (2000) 
Functional Insights from the Structure of the 30S Ribosomal Subunit and Its Interactions with Antibiotics. Nature, 407, 340-348. http://dx.doi.org/10.1038/35030019

[22] Bollen, B., Davies, J., Ozaki, M. and Mizushima, S. (1969) Ribosomal Protein Conferring Sensitivity to the Antibiotic Spectinomycin in Escherichia coli. Science, 165, 85-86. http://dx.doi.org/10.1126/science.165.3888.85

[23] Sigmund, C.D., Ettayebi, M. and Morgan, E.A. (1984) Antibiotic Resistance Mutations in 16S and 23S Ribosomal RNA Genes of Escherichia coli. Nucleic Acids Research, 12, 4653-4663. http://dx.doi.org/10.1093/nar/12.11.4653

[24] Garcia-Ortega, L., Alvarez-Garcia, E., Gavilanes, J.G., Martinez-del-Pozo, A. and Joseph, S. (2010) Cleavage of the Sarcin-Ricin Loop of 23S rRNA Differentially Affects EF-G and EF-Tu Binding. Nucleic Acids Research, 38, 41084119. http://dx.doi.org/10.1093/nar/gkq151

[25] Horan, L.H. and Noller, H.F. (2007) Intersubunit Movement Is Required for Ribosomal Translocation. Proceedings of the National Academy of Sciences of the United States of America, 104, 4881-4885. http://dx.doi.org/10.1073/pnas.0700762104

[26] Holbrook, S.R., Sussman, J.L., Warrant, R.W. and Kim, S.H. (1978) Crystal Structure of Yeast Phenylalanine Transfer RNA II. Structural Features and Functional Implications. Journal of Molecular Biology, 123, 631-660.

[27] Demeshkina, N., Jenner, L., Westhof, E., Yusupov, M. and Yusupova, G. (2012) A New Understanding of the Decoding Principle on the Ribosome. Nature, 484, 256-259. http://dx.doi.org/10.1038/nature10913

[28] Prince, J.B., Taylor, B.H., Thurlow, D.L., Ofengand, J. and Zimmermann, R.A. (1982) Covalent Crosslinking of $\mathrm{tRNA}_{1}{ }^{\text {Val }}$ to $16 \mathrm{~S}$ RNA at the Ribosomal P Site: Identification of Crosslinked Residues. Proceedings of the National Academy of Sciences of the United States of America, 79, 5450-5454. http://dx.doi.org/10.1073/pnas.79.18.5450

[29] Sprinzl, M., Wagner, T., Lorenz, S. and Erdmann, V.A. (1976) Regions of tRNA Important for Binding to the Ribosomal A and P Sites. Biochemistry, 15, 3031-3039. http://dx.doi.org/10.1021/bi00659a015

[30] Spahn, C.M. and Nierhaus, K.H. (1998) Models of the Elongation Cycle: An Evaluation. Biological Chemistry, 379, 753-772.

[31] Nagano, K. and Nagano, N. (1997) Transfer RNA Docking Pair Model in the Ribosomal Pre- and Post-Translocational States. Nucleic Acids Research, 25, 1254-1264. http://dx.doi.org/10.1093/nar/25.6.1254

[32] Ogle, J.M., Brodersen, D.E., Clemons Jr., W.M., Tarry, M.J., Carter, A.P. and Ramakrishnan, V. (2001) Recognition of Cognate Transfer RNA by the 30S Ribosomal Subunit. Science, 292, 897-902. http://dx.doi.org/10.1126/science.1060612

[33] Fredrick, K. and Noller, H.F. (2003) Catalysis of Ribosomal Translocation by Sparsomycin. Science, 300, $1159-1162$. http://dx.doi.org/10.1126/science.1084571

[34] Peske, F., Savelsbergh, A., Katunin, V.I., Rodnina, M.V. and Wintermeyer, W. (2004) Conformational Changes of the Small Ribosomal Subunit during Elongation Factor G-Dependent tRNA-mRNA Translocation. Journal of Molecular Biology, 343, 1183-1194. http://dx.doi.org/10.1016/j.jmb.2004.08.097

[35] Feinberg, J.S. and Joseph, S. (2001) Identification of Molecular Interactions between P-Site tRNA and the Ribosome Essential for Translocation. Proceedings of the National Academy of Sciences of the United States of America, 98, 1112011125. http://dx.doi.org/10.1073/pnas.211184098

[36] Schmeing, T.M., Voorhees, R.M., Kelley, A.C., Gao, Y.G., Murphy IV, F.V., Weir, J.R. and Ramakrishnan, V. (2009) The Crystal Structure of the Ribosome Bound to EF-Tu and Aminoacyl-tRNA. Science, 326, 688-694. http://dx.doi.org/10.1126/science.1179700

[37] Schmeing, T.M., Voorhees, R.M., Kelley, A.C. and Ramakrishnan, V. (2011) How Mutations in tRNA Distant from the Anticodon Affect the Fidelity of Decoding. Nature Structural \& Molecular Biology, 18, 432-436. http://dx.doi.org/10.1038/nsmb.2003

[38] AEvarsson, A., Brazhnikov, E., Garber, M., Zheltonosova, J., Chirgadze, Y., al-Karadaghi, S., et al. (1994) Three Dimensional Structure of the Ribosomal Translocase: Elongation Factor G from Thermus thermophilus. EMBO Journal, 13, 3669-3677.

[39] Gao, Y.G., Selmer, M., Dunham, C.M., Weixlbaumer, A., Kelley, A.C. and Ramakrishnan, V. (2009) The Structure of the Ribosome with Elongation Factor G Trapped in the Posttranslocational State. Science, 326, 694-699. http://dx.doi.org/10.1126/science.1179709

[40] Kisselev, L.L. and Buckingham, R.H. (2000) Translational Termination Comes of Age. Trends in Biochemical Sciences, 25, 561-566. http://dx.doi.org/10.1016/S0968-0004(00)01669-8

[41] Ito, K., Uno, M. and Nakamura, Y. (2000) A Tripeptide “Anticodon” Deciphers Stop in Messenger RNA. Nature, 403, 680-684.

[42] Rawat, U.B., Zavialov, A.V., Sengupta, J., Valle, M., Grassucci, R.A., Linde, J., et al. (2003) A Cryo-Electron Microscopic Study of Ribosome-Bound Termination Factor RF2. Nature, 421, 87-90. http://dx.doi.org/10.1038/nature01224 
[43] Klaholz, B.P., Pape, T., Zavialov, A.V., Myasnikov, A.G., Orlova, E.V., Vestergaard, B., et al. (2003) Structure of the Escherichia coli Ribosomal Termination Complex with Release Factor 2. Nature, 421, 90-94. http://dx.doi.org/10.1038/nature01225

[44] Song, H., Mugnier, P., Das, A.K., Webb, H.M., Evans, D.R., Tuite, M.F., et al. (2000) The Crystal Structure of Human Eukaryotic Release Factor eRF1-Mechanism of Stop Codon Recognition and Peptidyl-tRNA Hydrolysis. Cell, 100, 311-321. http://dx.doi.org/10.1016/S0092-8674(00)80667-4

[45] Osawa, S., Jukes, T.H., Watanabe, K. and Muto, A. (1992) Recent Evidence for Evolution of the Genetic Code. Microbiological Reviews, 56, 229-264.

[46] Nierhaus, K.H. (1990) The Allosteric Three-Site Model for the Ribosomal Elongation Cycle: Features and Future. Biochemistry, 29, 4997-5008. http://dx.doi.org/10.1021/bi00473a001

[47] Di Giacco, V., Marquez, Y., Qin, Y., Pech, M., Triana-Alonso, F.J., Wilson, D.N. and Nierhaus, K.H. (2011) Shine-Dalgarno Interaction Prevents Incorporation of Noncognate Amino Acids at Codon Following the AUG. Proceedings of the National Academy of Sciences of the United States of America, 108, 16980-16985.

[48] Geigenmüller, U. and Nierhaus, K.H. (1990) Significance of the Third tRNA Binding Site, the E-Site, on E. coli Ribosomes for the Accuracy of Translation: An Occupied E-Site Prevents the Binding of Non-Cognate Aminoacyl-tRNA to the A-Site. EMBO Journal, 9, 4527-4533.

[49] Schilling-Bartetzko, S., Bartetzko, A. and Nierhaus, K.H. (1992) Kinetic and Thermodynamic Parameters for tRNA Binding to the Ribosome and for the Translocation Reaction. Journal of Biological Chemistry, 267, 4703-4712.

[50] Nierhaus, K.H. (2006) Decoding Errors and the Involvement of the E-Site. Biochimie, 88, 1013-1019. http://dx.doi.org/10.1016/j.biochi.2006.02.009

[51] Semenkov, Y.P., Rodnina, M.V. and Wintermeyer, W. (1996) The “Allosteric Three-Site Model” of Elongation Cannot Be Confirmed in a Well-Defined Ribosome System from Escherichia coli. Proceedings of the National Academy of Sciences of the United States of America, 93, 12183-12188. http://dx.doi.org/10.1073/pnas.93.22.12183

[52] Uemura, S., Aitken, C.E., Korlach, J., Flusberg, B.A., Turner, S.W. and Puglisi, J.D. (2010) Real-Time tRNA Transit on Single Translating Ribosomes at Codon Resolution. Nature, 464, 1012-1017. http://dx.doi.org/10.1038/nature08925

[53] Chen, C., Stevens, B., Kaur, J., Smilansky, Z., Cooperman, B.S. and Goldman, Y.E. (2011) Allosteric vs. Spontaneous Exit-Site (E-Site) tRNA Dissociation Early in Protein Synthesis. Proceedings of the National Academy of Sciences of the United States of America, 108, 16980-16985. http://dx.doi.org/10.1073/pnas.1106999108

[54] Petropoulos, A.D. and Green, R. (2012) Further in Vitro Exploration Fails to Support the Allosteric Three-Site Model. Journal of Biological Chemistry, 287, 11642-11648. http://dx.doi.org/10.1074/jbc.C111.330068

[55] Liao, P.Y., Gupta, P., Petrov, A.N., Dinman, J.D. and Lee, K.H. (2008) A New Kinetic Model Reveals the Synergistic Effect of E-, P- and A-Sites on +1 Ribosomal Frameshifting. Nucleic Acids Research, 36, 2619-2629. http://dx.doi.org/10.1093/nar/gkn100

[56] Sundararajan, A., Michaud, W.A., Qian, Q., Stahl, G. and Farabaugh, P.J. (1999) Near-Cognate Peptidyl-tRNAs Promote +1 Programmed Translational Frameshifting in Yeast. Molecular Cell, 4, 1005-1015. http://dx.doi.org/10.1016/S1097-2765(00)80229-4

[57] Bartetzko, A. and Nierhaus, K.H. (1988) $\mathrm{Mg}^{2+} / \mathrm{NH}^{4+} /$ Polyamine System for Polyuridine-Dependent Polyphenylalanine Synthesis with Near in Vivo Characteristics. Methods in Enzymology, 164, 650-658. http://dx.doi.org/10.1016/S0076-6879(88)64075-4

[58] Bernstein, H.J. (2000) Recent Changes of RasMol, Recombining the Variants. Trends in Biochemical Sciences, 25, 453-455. http://dx.doi.org/10.1016/S0968-0004(00)01606-6 